\title{
An Inductively Coupled Ultra-Thin, Flexible, and Passive RF Resonator for MRI Marking and Guiding Purposes: Clinical Feasibility
}

\author{
Akbar Alipour (10 ${ }^{1}$ Sayim Gokyar, ${ }^{1}$ Oktay Algin, ${ }^{1,2}$ Ergin Atalar, ${ }^{1}$ \\ and Hilmi Volkan Demir ${ }^{1,3}$ *
}

Purpose: The purpose of this study is to develop a wireless, flexible, ultra-thin, and passive radiofrequency-based MRI resonant fiducial marker, and to validate its feasibility in a phantom model and several body regions.

Methods: Standard microfabrication processing was used to fabricate the resonant marker. The proposed marker consists of two metal traces in the shape of a square with an edge length of $8 \mathrm{~mm}$, with upper and lower traces connected to each other by a metalized via. A 3T MRI fiducial marking procedure was tested in phantom and ex vivo, and then the marker's performance was evaluated in an MRI experiment using humans. The radiofrequency safety was also tested using temperature sensors in the proximity of the resonator.

Results: A flexible resonator with a thickness of $115 \mu \mathrm{m}$ and a dimension of $8 \times 8 \mathrm{~mm}$ was obtained. The experimental results in the phantom show that at low background flip angles $\left(6-18^{\circ}\right)$, the resonant marker enables precise and rapid visibility, with high marker-to-background contrast and signal-to-noise ratio improvement of greater than 10 in the vicinity of the marker. Temperature analysis showed a specific absorption ratio gain of 1.3. Clinical studies further showed a successful biopsy procedure using the fiducial marking functionality of our device.

Conclusions: The ultra-thin and flexible structure of this wireless flexible radiofrequency resonant marker offers effective and safe MR visualization with high feasibility for anatomic marking and guiding at various regions of the body. Magn Reson Med 80:361-370, 2018. (C) 2017 International Society for Magnetic Resonance in Medicine.

Key words: inductive coupling; MR-guided marker; RF-passive resonator; thin-film structure
${ }^{1}$ Department of Electrical and Electronics Engineering, National Magnetic Resonance Research Center (UMRAM) National Nanotechnology Research Center and Institute of Material Science and Nanotechnology (UNAM) Department of Physics, Bilkent University, Bilkent, Ankara, Turkey.

${ }^{2}$ Department of Radiology, Ankara Ataturk Training and Research Hospital, Ankara, Turkey.

${ }^{3}$ LUMINOUS! Center of Excellence for Semiconductor Lighting and Displays, School of Electrical and Electronic Engineering, School of Mathematical and Physical Sciences, Nanyang Technological University, Singapore.

${ }^{*}$ Correspondence to: Hilmi Volkan Demir, Ph.D., Department of Electrical and Electronics Engineering, Bilkent University, Bilkent, 06800, Ankara, Turkey. E-mail: volkan@bilkent.edu.tr

Received 10 February 2017; revised 17 September 2017; accepted 15 October 2017

DOI 10.1002/mrm.26996

Published online 16 November 2017 in Wiley Online Library (wileyonlinelibrary.com).

(C) 2017 International Society for Magnetic Resonance in Medicine

\section{INTRODUCTION}

Anatomical position marking for interventional guidance is a major challenge and a significant problem for MRI. Solutions to this problem normally require an MR-visible object that precisely presents a reference location as well as real-time visualization (1-3). Magnetic resonance-visible markers are not only one of the most preferred tools for referencing purposes, but have also gained considerable interest as a modality for guiding medical instruments, such as biopsy needles, for minimally invasive interventions $(4,5)$. The basic principle of MR-visible markers relies on introducing special contrast with respect to the morphological MR image $(6,7)$. Intrinsic artifacts of MRI-guided needles or the tracking device were the foundation of the earlier fiducial marking techniques (8-10). Recently, efforts have focused primarily on the MRI visibility of these fiducial marker devices and detectability improvement (11-15). Moreover, the identification and marking of MR-visible objects have introduced possible solutions to motion artifact reduction (16) and automatic 3-dimensional (3D) localization $(17,18)$.

According to their structure and detection technique, the MR-visible markers can be classified as material-based markers or radiofrequency (RF) based markers. The material-based markers are either paramagnetic materials, which change the local signal by local field distortion $(19,20)$, or contrast agents, which shorten $T_{1}$ or $T_{2}$ values of the tissue and alter the MR signal intensity locally (21-23). Although this technique avoids the possible safety hazards of RF, the material-based markers are not visible under all imaging parameters (24). The RF-based markers can also be categorized into two types: active RFbased markers and passive RF-based markers. The active techniques use small pick-up RF coils that are connected to the MR scanner through an electrically conductive wire. Fast and accurate positioning can be obtained using multiple active markers connected to the separate receiver channels. However, a possible safety hazard is introduced by the electric coupling between the long connecting wires and transmitter coils (25-28). Furthermore, the interface with the receiver and crosstalk between the wires are further significant problems. These problems limit the clinical implementation of the active design (29-31). To simplify the clinical implementation of the RF-based markers and to avoid their possible safety hazards, passive RF-based markers based on inductively coupled RF coils have been examined (32-34). These wireless, passive RFbased markers use simple resonant LC circuits that are 
made of lumped electrical components, including RF coils and chip-scale nonmagnetic capacitors. These markers do not need any elongated conductors to carry the RF signal. The signal is transmitted and received by inductive coupling to standard transmitter/receiver MR coils. The MR visibility of these markers results from local MR signal amplification through flip angle (FA) enhancement during the RF excitation, and signal enhancement during the signal acquisition, which leads to special positive contrast. Consequently, higher marker-to-background contrast is achieved at very low FAs. Because of the requirement for quick position detection, until now, studies have been reported to use passive RF-based markers that provide robust localization. However, the size and rigid structure of these passive markers limit their use for marking purposes to critical regions of the body (e.g., cerebral cortex). To overcome these disadvantages, we developed a flexible, ultra-thin, and surface-mountable wireless passive RFbased resonator marker. This proposed marker consists of two conducting lines deposited on both sides of its dielectric substrate, ensuring distributed capacitance along the conductive lines, and the upper and lower rings are connected to each other by a via through the substrate (35-37).

In this work, we test and validate the clinical feasibility of anatomic-position marking using our proposed passive RF-based marker. The ultra-thin structure and flexibility of this device allow for an opportunity to demonstrate fiducial marking at various regions of the body. Here, the characteristics of the developed marker were evaluated to enable precise and rapid positioning of the marker for biopsy needle guidance under clinical MR imaging. The marker's performance was evaluated in a 3T MRI scanner on a phantom model, an ex vivo animal model, and in the clinic.

\section{METHODS}

\section{Wireless, Passive RF-Based Resonator Marker}

We designed an ultra-thin, flexible, and passive RF-based resonator to be used as an MR-guided marker. Our design consists of a resonant helical structure with incorporated layered capacitors to achieve resonance. The developed passive marker has two significant components, namely, a distributed inductor introduced as a passive power source in the form of conductive paths, and a distributed capacitor, which is a dielectric layer sandwiched between two conductive paths (Figs. 1a-1c). Our proposed device architecture is a multilayer laminated structure consisting of two broadsidecoupled split-ring resonators, in which the upper and lower rings are connected to each other by a metalized via. Standard microfabrication technique was used to pattern $3-\mu \mathrm{m}$ thick gold $(\mathrm{Au})$ layers on both sides of the $7-\mu \mathrm{m}$-thick polyimide film (Kapton HN, DuPont, Berlin, Germany) with a dielectric constant of 3.4. The thin-film resonant marker has a square-shaped double-turn coil design with a line length of $8 \mathrm{~mm}$ and a conductor width of $1 \mathrm{~mm}$ (Figs. 1d and 1e). To obtain electrical isolation and biocompatibility, and to avoid the capacitive effects resulting from interactions between the resonator marker and the surrounding tissue, the resonator was coated with a $50-\mu \mathrm{m}$-thick polydimethylsiloxane (Dow Corning Sylgard 184 Silicone Elastomer, Sigma-Aldrich, Munich, Germany) layer on both sides. Polydimethylsiloxane is a commonly used polymer for microelectromechanical systems that is extensively applied as a biocompatible material for implanted structures (38).

The designed structure can be described as an LC circuit, in which the equivalent lumped circuit elements ( $\mathrm{L}$ and $\mathrm{C}$ ) are arranged by the thickness and electrical properties of the dielectric substrate, the width of the conductor line, and the conductivity of the deposited conductor layer.

The operating frequency of the resonator is sensitive to the dielectric thickness, such that $10 \%$ error in thickness results in 5\% shift in the resonance frequency. To tune the small structure, a large distributed capacitance was used.

\section{Marker Characterization}

To characterize the MRI properties of our resonator structure, test resonator markers were fabricated to assess the resonator's characteristics. To this end, the reflection coefficient $\left(S_{11}\right)$ of a pick-up coil that is weakly coupled to the resonator was measured using a network analyzer (Agilent E5061A, Santa Clara, CA, USA). The characterizations for the ultimate device were achieved with the resonator marker and the pick-up coil immersed in a liquid saline phantom. The passive resonator marker was designed to work in the resonance frequency of a 3T MR scanner (Magnetom Trio, Siemens, Munich, Germany, $f_{o}=123 \mathrm{MHz}$ ).

The characteristics of the passive RF resonator marker were evaluated using a full electromagnetic numerical simulation (CST Microwave Studio, Darmstadt, Germany) and analytical methods. The simulation setup contained a resonator marker positioned perpendicular to the static magnetic field (i.e., the planar resonator normal is perpendicular to $\mathrm{B}_{0}$ ) that was set over the center of the lateral surface of a cylindrical saline phantom (22-cm diameter and $15 \mathrm{~cm}$ in height; dielectric constant: 70; conductivity: $0.65 \mathrm{~s} / \mathrm{m})$. The measurement was conducted for the loaded and unloaded (free space) cases. In the analytical method, the resistance $(R=0.42 \Omega)$, inductance $(L=60 \mathrm{nH})$, and capacitance $(\mathrm{C}=8.71 \mathrm{nF})$ values for the unloaded resonator marker tuned to $123 \mathrm{MHz}$ were calculated (35).

\section{Phantom MR Experiment}

Image acquisition was performed using a $3 \mathrm{~T}$ MR scanner (Tim Trio, Siemens, Erlangen, Germany) on a standard saline phantom. The phantom was excited by the body RF coil, whereas the signal reception was performed by a 12-channel head coil (3T Head Matrix A Tim Coil, Siemens, Erlangen, Germany). The MR images were acquired using gradient-echo (GRE) sequences. According to these image groups, the signal-to-noise ratio (SNR) analysis was performed to determine the signal homogeneity and penetration depth. The SNR profiles are computed using the following formula (39):

$$
S N R=\frac{S_{a v r}}{\sqrt{2 N\left(\sigma_{n}^{2}+m_{n}^{2}\right)}}
$$

where $S_{a v r}$ is the averaged signal intensity, $N$ is the number of array coils; and $\sigma_{n}$ and $m_{n}$ are the standard deviation and mean value of noise, respectively.

The sequence parameters for the phantom experiment were as follows: repetition time (TR) $=300 \mathrm{~ms}$, echo time 
FIG. 1. Three-dimensional schematic (a), top view (b), and front view (c) of the architectural construction of our passive RFresonator marker (not drawn to scale). The device structure consists of two metal-layer split-ring resonators that are patterned on both sides of a Kapton dielectric substrate, where layers are connected to each other through via metallization. b, c: Array of $8 \times 8 \mathrm{~mm}$ resonator markers fabricated on a 7 - $\mu \mathrm{m}$-thick flexible Kapton film.
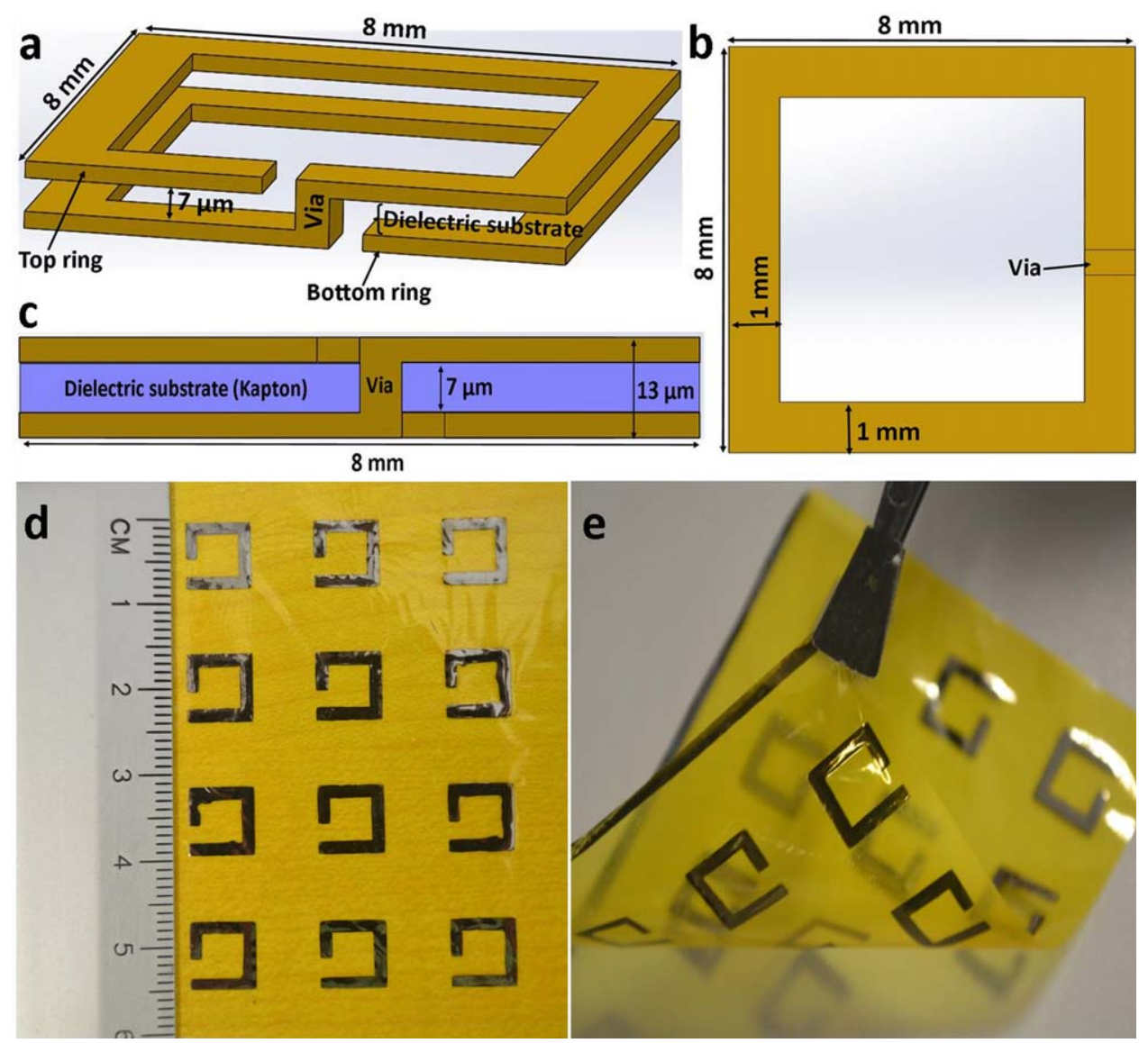

$(\mathrm{TE})=10 \mathrm{~ms}$, matrix size $=256 \times 256$, slice thickness $=$ $3 \mathrm{~mm}$, and field of view $(\mathrm{FOV})=220 \times 220 \mathrm{~mm}^{2}$.

$\mathrm{A} \mathrm{B}_{1}^{+}(+)$map was calculated inside the phantom to determine the $\mathrm{B}_{1}$ field distribution in the vicinity of the resonator marker. The induced current on the resonator results in $B_{1}$ field amplification at the proximity of the resonator marker that consequently magnifies the FA (31). The generated linearly polarized magnetic field $B_{R}$ can be decomposed into right and left rotating polarized components (34).

$$
\mathrm{B}_{\mathrm{R}}(\mathrm{t})=\mathrm{B}_{\mathrm{RR}}(\mathrm{t})+\mathrm{B}_{\mathrm{RL}}(\mathrm{t})
$$

where $B_{R R}(t)$ and $B_{R L}(t)$ are the right and left circularly polarized field components, respectively. The excited spins in MRI generate a rotating magnetic field that can be picked up by a forward-polarized receiving coil; therefore, only the left circularly polarized field component, $\mathrm{B}_{\mathrm{RL}}(\mathrm{t})$, contributes to the $\mathrm{B}_{1}^{+}(+)$map. A double FA $\mathrm{B}_{1}^{+}(+)$map (40) (3 T, spin echo, TR/TE $=3000 / 12 \mathrm{~ms}$, matrix size $=256 \times 256$, slice thickness $=2 \mathrm{~mm}$ ) was obtained in the phantom, which included a resonator marker. The applied FA needed to obtain the Ernst angle in the vicinity of the resonator, $\alpha_{\mathrm{ER}}$, is 1/Q of the Ernst angle for the phantom in the absence of the resonator, where $\mathrm{Q}$ is the quality factor of the resonator (11).

The coupling of the transmitted RF field and the resonator marker is directly related to the resonator's orientation. The transmitted field can be decomposed into components that are perpendicular and transverse to the surface-normal vector of the resonator. Only the transverse component will contribute to the effective received signal. Therefore, to achieve the highest performance, the surface-normal vector of the resonator marker must be aligned perpendicular to the static magnetic field.

\section{Orientation Dependency}

The resonator's maximum coupling with the RF body coil when its normal axis is perpendicular to the main magnetic field and the coupling is reduced to zero is when the normal axis of the resonator is parallel with $\mathrm{B}_{0}$. To demonstrate this effect, the marker orientation was tested with a phantom experiment. The resonator alignment has changed from $0^{\circ}$ to $90^{\circ}$ in steps of $5^{\circ}$. The sensitivity of the signal intensity to the resonator's orientation with regard to the alignment of the main magnetic field was evaluated by calculating the mean signal intensity in the region of interest.

\section{Radiofrequency Safety Test}

The proposed structure is not disabled during RF excitation, so it may pose a hazard to the patient as a result of RF heating and associated temperature increases in the tissue. Temperature analysis throughout the RF excitation was realized with our passive resonator marker tuned to the loaded resonance frequency. The resonator marker was immersed in a cylindrical gel phantom $40 \mathrm{~cm}$ in diameter and $25 \mathrm{~cm}$ height (heat resistivity $=0.661 \mathrm{~W} / \mathrm{mK}$, heat capacity $=4454 \mathrm{~J} /(\mathrm{kg} \cdot \mathrm{K})$, and diffusivity $\left.=1.48 \mathrm{~mm}^{2} / \mathrm{s}\right)$. We 
used electromagnetic computer simulation technology to identify the possible highest specific absorption rate (SAR) regions. Four fiber-optic probes $\left(\mathrm{P}_{1}-\mathrm{P}_{4}\right)$ of an optic thermometer sensor (Neoptix RF-04-1, Québec, Canada) were set inside the phantom near the resonator in locations that were predicted to show the maximum heat. The fifth probe, $\mathrm{P}_{5}$, was used to collect the temperature of a reference point inside the phantom. The assembly was placed in the isocenter of the body coil (41). The phantom with resonator inside was subjected to a high SAR sequence (GRE: $\mathrm{TR}=2.6 \mathrm{~ms}$, $\mathrm{TE}=1.5 \mathrm{~ms}, \mathrm{FA}=50^{\circ}$, slice thickness $=200 \mathrm{~mm}, \quad$ slice number $=2, \quad \mathrm{FOV}=220 \times$ $220 \mathrm{~mm}^{2}$, average $=32$ ). The sequence was continuously run for $16 \mathrm{~min}$ at the field strength of $14 \mu \mathrm{T}$. The reported whole-body average SAR by the scanner was $2 \mathrm{~W} / \mathrm{kg}$. Because the scanner parameters were calculated for human experiment, the reported SAR by the scanner may not reflect the exact value of the applied SAR into the phantom. In compliance with the ASTM standard test method F2182-11a (42), we calculated the SAR value based on the temperature measurements instead of the reported SAR by the scanner. The SAR was calculated using

$$
\mathrm{SAR}=\mathrm{C} \frac{d T}{d t}
$$

where $C$ is the heat capacity, $T$ is the temperature, and $t$ is the time. The SAR gain was calculated by dividing the maximum temperature increase measured from the temperature probes by the temperature increase at the reference probe $\left(\mathrm{P}_{5}\right)$.

\section{Marking Under MR Experiment}

Ex vivo animal experiments were performed on the head of an adult sheep cadaver. The aim of this experiment was to show the feasibility of placing our proposed passive resonant marker over the cerebral cortex, and in addition, to visualize the markers, the needle, and the brain anatomy simultaneously in a single MRI image. For this purpose, an array of markers $(4 \times 5)$ was placed onto the cerebral cortex of the cadaver. The cadaver head with an array of markers was positioned inside the head coil in a $3 \mathrm{~T}$ MR scanner. Imaging was realized by a GRE sequence $(\mathrm{TR}=750 \mathrm{~ms}, \mathrm{TE}=20 \mathrm{~ms}$, matrix $\operatorname{size}=256 \times$ 256, $\quad \mathrm{FA}=15^{\circ}, \quad$ slice thickness $=3 \mathrm{~mm}, \quad \mathrm{FOV}=160 \times$ $160 \mathrm{~mm}^{2}$, bandwidth $=260 \mathrm{~Hz} / \mathrm{Px}$ ) using the body coil as a transmitter and the 12-channel head coil as a receiver. The transverse and sagittal MR images of the brain were obtained, of which the intersection of these two images represented useful information for any possible biopsy procedure. The contrast of the marker with respect to the morphological MR images allowed the location of the markers on the surface of the brain to be identified.

Human experiments were performed on a volunteer in accordance with a protocol approved by the institutional review board. The performance of the wireless, passive RF-based markers was studied on the forehead and the knee. For the head scanning, the volunteer with the aligned markers on his or her forehead was placed inside a $3 \mathrm{~T}$ MR scanner, where the body RF coil was used as a transmitter and a 12-channel head coil as a receiver. The knee was scanned using a 3T 15-channel transmit/ receive coil ( $\mathrm{Tx} / \mathrm{Rx}$, Quality Electrodynamics (QED), Mayfield Village, OH, USA). The same imaging protocol as in the ex vivo experiments was applied for the human imaging.

Clinical study of the proposed resonator markers was performed on a patient with a tumor mass in their brain. After the patient was positioned on the scanner table, an array of the resonator markers $(2 \times 2)$ was fixed onto the head of the patient. The position of the resonator markers with respect to the mass was confirmed using $\mathrm{T}_{1}$-weighted GRE acquisition $(\mathrm{TR}=100 \mathrm{~ms}, \mathrm{TE}=3.1 \mathrm{~ms}$, matrix size $=256 \times 256, \mathrm{FA}=20^{\circ}$, slice thickness $=3 \mathrm{~mm}$, FOV $=160 \times 160 \mathrm{~mm}^{2}$, bandwidth $=260 \mathrm{~Hz} / \mathrm{Px}$ ). After the acquisition of coronal and sagittal plane images, the patient table was withdrawn from the scanner. The point for insertion of the biopsy needle was selected based on the position of the resonator markers. The needle was inserted into the brain by confirming the needle trajectory with two intraoperative scans, as described in (43).

\section{RESULTS}

\section{Marker Characterization}

The characteristics of the resonator marker were evaluated on both unloaded and loaded cases. The measured frequency responses of the resonator marker at the module of reflection coefficient showed that loading effect shifted the resonance frequency down from 123.3 to $121.4 \mathrm{MHz}$ (Fig. 2). To compensate for this frequency shift, the marker's operating frequency was tuned to higher frequency in free space, and this was shifted down to the scanner's Larmor frequency by loading effect.

\section{Effect of the Marker in the Phantom}

Figure 3 reveals the feasibility of coronal and transverse imaging of the wireless resonator marker with corresponding SNR profiles. The marker is visible for an approximate 4-mm-depth penetration in the transverse plane (Fig. 3a) and approximate $8 \times 8 \mathrm{~mm}$ surface area in the coronal plane (Fig. 3b). Inductive coupling between the resonator marker and surrounding medium leads to local signal enhancement in the vicinity of the marker. Consequently, a high-intensity signal is detected from the medium that is influenced by the marker. Strong sensitivity enhancement at the center of the marker is indicated by a sharp peak in the SNR profile of both the coronal and transverse images (Figs. 3c and 3d). The SNR profile in the transverse plane shows that by moving away from the center of the marker, the signal enhancement is decreased.

$B_{1}$ map analysis shows the spatial distribution of the RF magnetic field in the phantom, including the resonator marker (Fig. 4). The field generated by the resonator results in FA amplification around the resonator, and this enhancement is diminished by moving away from the center of the resonator. The null $\mathrm{B}_{1}$ field at some regions around the resonator is the result of cancelation of the total $B_{1}$ field generated by the excitation RF field and the resonator field, which is shown as dark regions 


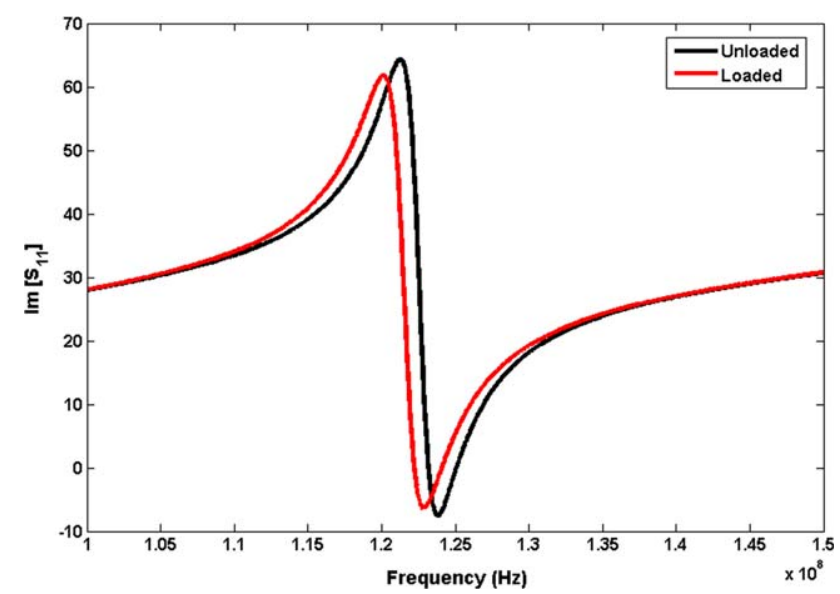

FIG. 2. Measured reflection coefficient of the resonator marker in loaded and unloaded states. Loading can affect the electrical characteristics of the resonator, such as shifting down the operating frequency.

in Figure 3b. A maximum FA gain of approximately 2.3 was obtained in the close vicinity of the resonator.

With the passive resonator marker immersed in the saline phantom, imaging with low FA in the range of $6^{\circ}$ to $18^{\circ}$ led to high signal intensity and consequently acceptable marker-to-background contrast. An excitation angle of $8^{\circ}$ presented the highest marker signal intensity. The maximum signal intensity occurred at a background FA of $82^{\circ}$ for the without-resonator case, which is in good agreement with the calculated Ernst angle $\left(\alpha_{E}=76^{\circ}\right)$. The ratio of background FAs $\left(82^{\circ} / 8^{\circ} \approx 10.2\right)$ that resulted in maximum signal in both the withoutand with-resonator cases corresponds to the resonator Qfactor. Increasing the background FAs results in a decrease of the resonator signal intensity in its vicinity.

\section{Orientation Variation}

The orientation dependence of the resonator can be seen in Figure 5. When the axis of the resonator is perpendicular to the main magnetic field, which is the resonator axis is in the transverse plane $\left(\theta=0^{\circ}\right)$, the coupling is maximum. As expected, the minimum signal intensity was obtained when the resonator's axis is in parallel with the main magnetic field $\left(\theta=90^{\circ}\right)$.

These results show that partial coupling between the marker and RF excitation (when the marker is tilted off its axis) can still provide signal enhancement to improve the SNR.
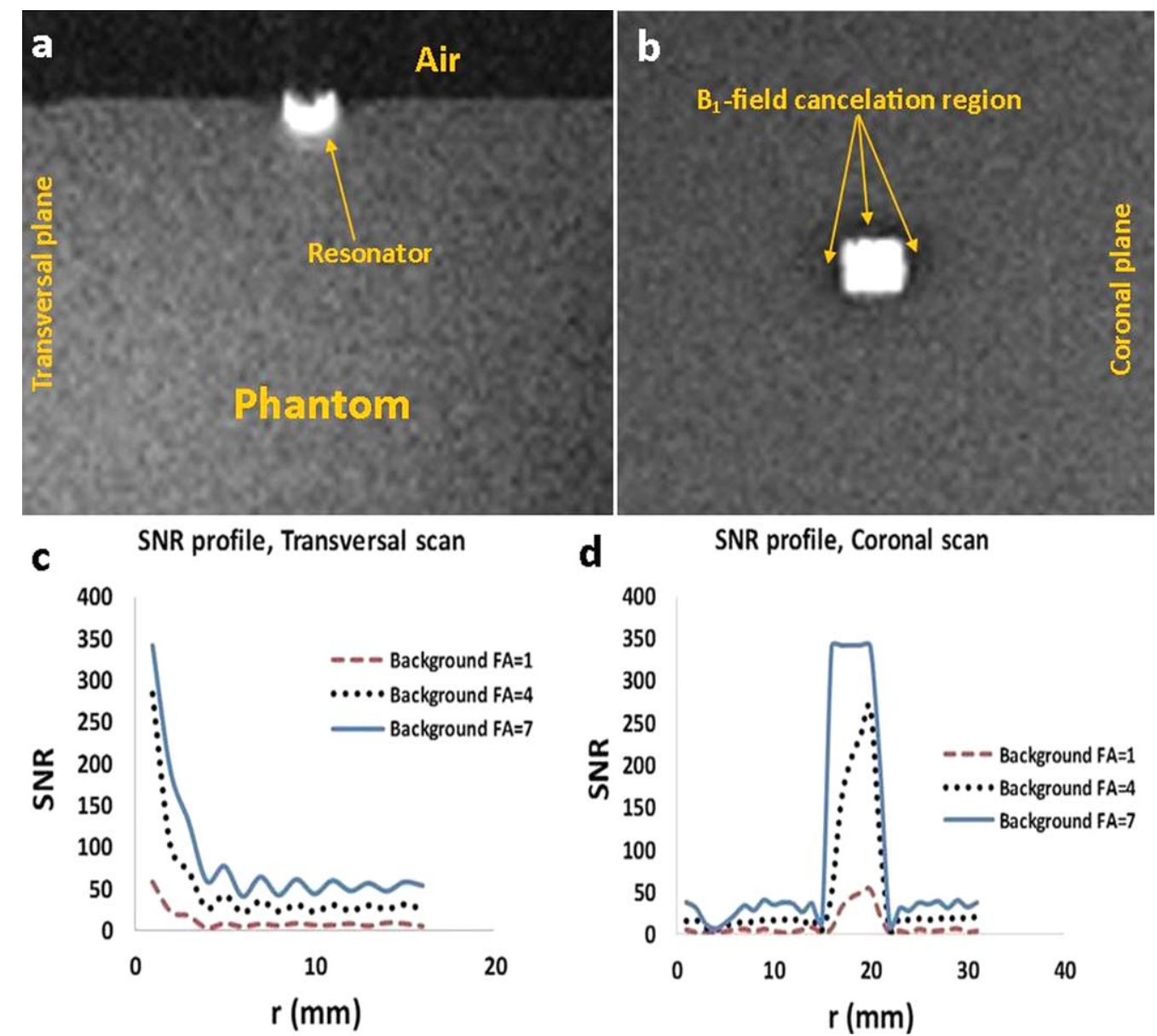

d

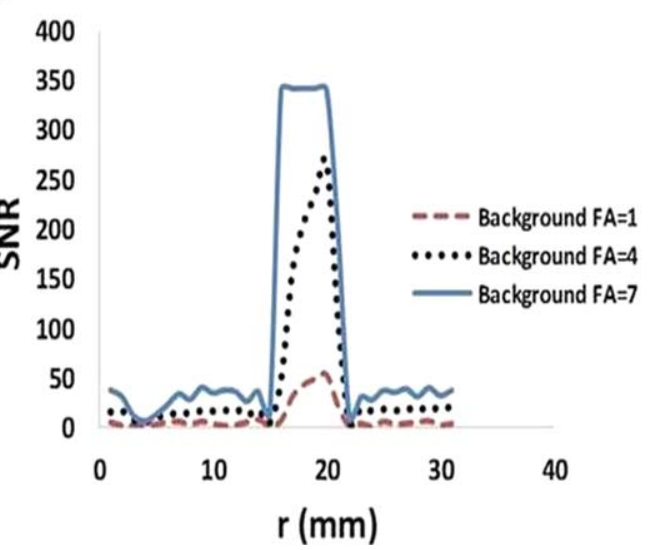

FIG. 3. a, b: Transverse and coronal low-FA GRE images of $8 \times 8 \mathrm{~mm}$ resonator markers. The markers are placed in the center of the lateral surface of a cylindrical saline phantom. The bright spot indicates the effect of the marker in local signal amplification, which leads to high marker-to-background contrast at very low excitation angle. c, d: Cross section of SNR profile through the middle marker shown in transverse scan (a) and in coronal scan (b) at three different background FAs. 

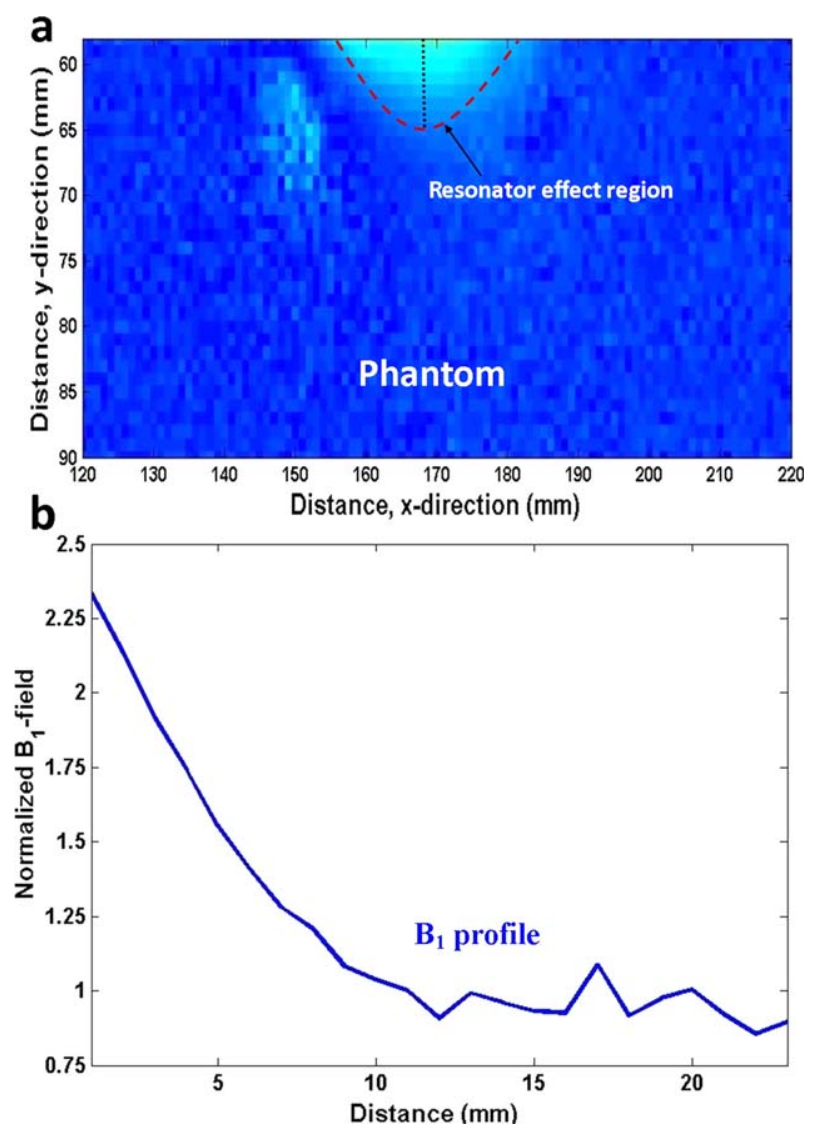

FIG. 4. a: $B_{1}$ field distribution of a phantom with the resonator marker inside. Double-angle method is used to analyze the $B_{1}$ field distribution and the effect of the resonator. The field generated by the resonator enhances the FA within close proximity. $\mathbf{b}$ : Plotted FA profile (normalized to background phantom $B_{1}$ value) shows maximum FA amplification of 2.3.

\section{Radiofrequency Safety}

The computer simulation technology was performed to calculate the SAR of the phantom close to the resonator. A SAR gain of 1.4 was reported in the proximity of the resonator (Fig. 6a). After estimating the maximum SAR regions, temperature sensors were positioned at the related points around the resonator (Fig. 6b). The RF heating measurements of the wireless resonator marker were performed under the GRE-based heating pulse sequence $\left(\mathrm{TR}=2.6 \mathrm{~ms}, \mathrm{TE}=1.5 \mathrm{~ms}, \mathrm{FA}=50^{\circ}\right.$, slice thickness $=$ $200 \mathrm{~mm}$, slice number $=2, \mathrm{FOV}=220 \times 220 \mathrm{~mm}^{2}$, average $=$ 32 ). All of the sensors experienced similar values of temperature increases $\left(\mathrm{P}_{1}=2.1^{\circ} \mathrm{C}, \quad \mathrm{P}_{2}=1.7^{\circ} \mathrm{C}, \quad \mathrm{P}_{3}=1.8^{\circ} \mathrm{C}\right.$, $\mathrm{P}_{4}=1.7^{\circ} \mathrm{C}$, and $\mathrm{P}_{5}=1.6^{\circ} \mathrm{C}$ ) (Fig. 6c). The highest temperature increase of $2.1^{\circ} \mathrm{C}$ was measured at point $\mathrm{P}_{1}$. This can be explained by the homogeneous electric-field distribution of the resonator within the phantom. According to the simulation and experimental results, we reported the highest SAR gain of 1.3. The maximum SAR gain of 1.3 was calculated by dividing the temperature increase measured at point $\mathrm{P}_{1}$ by the temperature increase at the reference point, $\mathrm{P}_{5}$ (Fig. 6c). To safely scan the patients with these markers, we recommend that the maximum allowed peak SAR values to be reduced by this factor.

\section{Anatomical MRI Experiments}

Figure 7a shows a photo of the resonators set over the cerebral cortex. Figures 7b and 7c display successful transverse and sagittal $\mathrm{T}_{2}^{*}$-weighted marker visualization of the resonator in the cadaver cerebral cortex, respectively.

In human experiments, the resonator markers appeared as bright spots in the $\mathrm{T}_{2}^{*}$-weighted GRE images of the head and knee (Figs. 8a and 8b).

In interventional study, the resonator markers fixed over the head of the patient provided a robust localization signal that showed as bright spots in MR images with respect to the background anatomy (Fig. 9a). The images were obtained on both the coronal and sagittal planes. The position of the tumor mass, which is shown to be surrounded by a white circle in Figure 9, is identified with respect to the resonators on these images. The intersection of the two imaging planes represented the point of insertion of the biopsy needle (Fig. 9b).

\section{DISCUSSION}

In this paper, we demonstrated clinical applications of our MR-visible, passive, RF-based resonator marker that was fabricated using standard microfabrication technique. Gold metallization was performed on both sides of the flexible dielectric substrate. The dielectric sandwiched between two conductive layers ensured distributive capacitance between these layers. This capacitive region is the main parameter in resonance-frequency tuning. The applied methodology consisted of a passive RF resonator circuit that inductively coupled to the RF receiver coils to eliminate any need for an external source.

The proposed MR-visible structure introduced an ultra-thin, flexible, and passive resonator marker (total thickness of $115 \mu \mathrm{m}$ and dimensions of $8 \times 8 \mathrm{~mm}$ ) with various inherent benefits. Our device was based on a self-resonating structure and used distributed components (inductor and capacitor) to operate at the Larmor frequency to provide more visualization of different surfaces of the body. In comparison with other standard markers, such as short- $\mathrm{T}_{1}$ (liquid) component-filled capsules, our proposed markers can be easily implemented

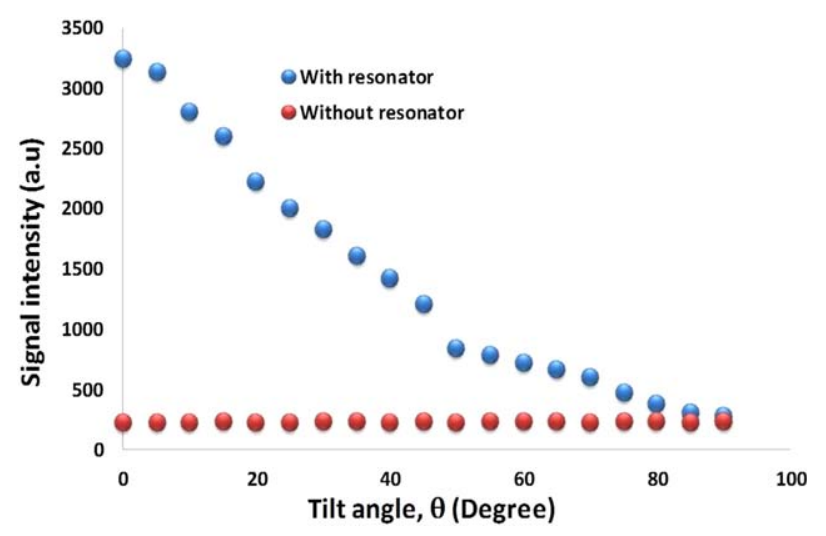

FIG. 5. Coupling dependence of signal intensity on the marker tilt angle $(\theta)$. With a GRE pulse sequence, the marker could be identified for a tilt angle up to $80^{\circ}$. 
FIG. 6. a: Electromagnetic computer simulation technology is conducted to predict the highest heating places. $P_{1}$ to $P_{5}$ indicate the location of the temperature probes for heat measurement. b: Three-dimensional schematic of the assembly for computing the local SAR. Photograph of the resonator with temperature sensors shows the arrangement of the probes near the resonator. $P_{1}$ is placed near the via. $P_{2}$ is set close to the center point, and $\mathrm{P}_{3}$ and $\mathrm{P}_{4}$ are placed at the inner and outer side of the resonator. $\mathrm{P}_{5}$ is set at another corner of the phantom, far from the resonator marker, as a reference sensor. c: Temperature recording from probes $P_{1}$ to $P_{5}$. Temperature rises during $16-\mathrm{min}$ $\mathrm{RF}$ exposure in a continuous high SAR pulse sequence. No significant temperature increases were reported over $16 \mathrm{~min}$. Temperature data from reference-point $P_{5}$ show a $1.6 \mathrm{C}^{\circ}$ increase. Maximum temperature increase is reported at $\mathrm{P}_{1}\left(2.1 \mathrm{C}^{\circ}\right)$, which corresponds to an SAR gain of 1.3.

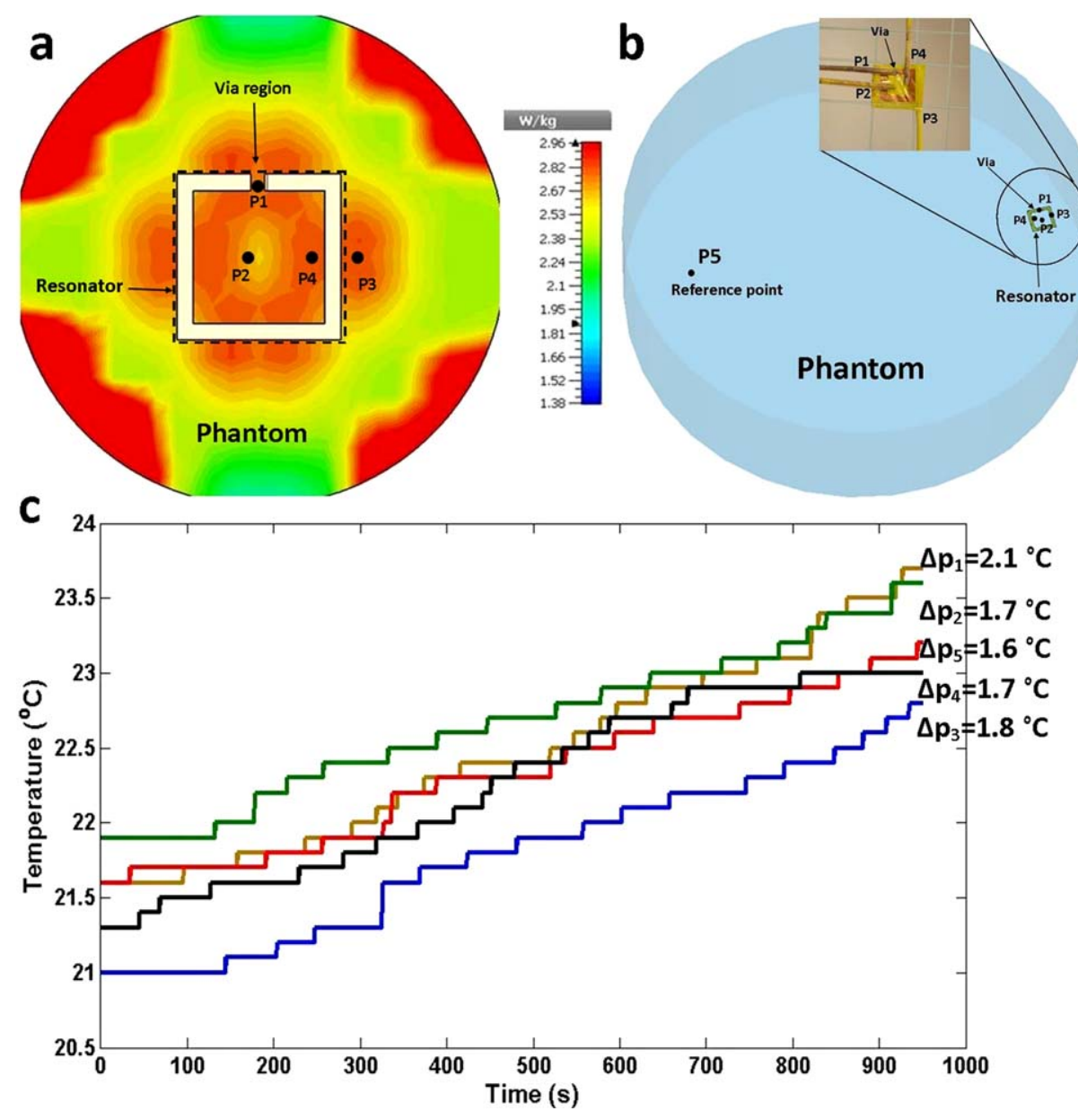

over various parts of the body-especially on critical regions such as the cerebral cortex. In addition, they provide higher marker-to-background contrast, which allows them to be clearly visible with respect to the anatomical background. Additionally, the device was coated with a thin layer of polydimethylsiloxane, which allows sterilization. In comparison with active markers, our resonant marker provides high SNR using routine pulse sequences without any need to manipulate the magnetic-field gradient pulses (44). As a result of being flexible, passive, and having ultra-thin structure properties, an array of the resonant markers can be easily set over the targeted area, whereas crosstalk between the cables is the main problem in active devices. However, low SNR condition when the marker is decoupled from the RF excitation is the major disadvantage of our designed resonant marker.

$\mathrm{B}_{1}$ map analysis results revealed a predominant contribution of the resonator marker to effective FA amplification of 2.3. The resonator amplified the effective FA, consequently leading to local SNR enhancement of greater than 10 in the proximity of the marker, with small background FAs $\left(6^{\circ}-18^{\circ}\right)$. The phantom signal variation for both without- and with-resonator cases was determined by altering the background FA. At small FAs, the resonator signal was more dominant. Increasing the FA ensured simultaneous visualization of the resonator and surrounding medium.
In our design, the two layers of the ring were connected to each other using a metalized via. One might have omitted this and used a structure similar to a splitring resonator (36); however, reducing the resonance frequency for a small structure may require larger capacitance than what we used in our design.

One of the significant safety concerns in our resonant markers implementation is the possibility of local heating hazards caused by inductive coupling during $\mathrm{RF}$ excitation. Our proposed passive RF resonator structure uses distributed components, which resulted in more evenly distributed power dissipation in comparison with lumped elements. Furthermore, homogeneous electric field distribution of the resonator resulted in a small number of hot spots and a small amount of heating in the vicinity of the resonator. The maximum SAR gain of 1.3 was reported in the proximity of the resonator inside a phantom. The SAR gain of the resonant marker can be further reduced by decoupling the resonator from the RF excitation. For example, thin-film back-toback diodes can be added to the current structure for this purpose.

An additional advantage of this resonator marker is weak sensitivity to orientation dependency. The device displayed maximum signal sensitivity when the surface normal vector is perpendicular to the static magnetic field $\left(\theta=0^{\circ}\right)$. This is a necessary condition to achieve 

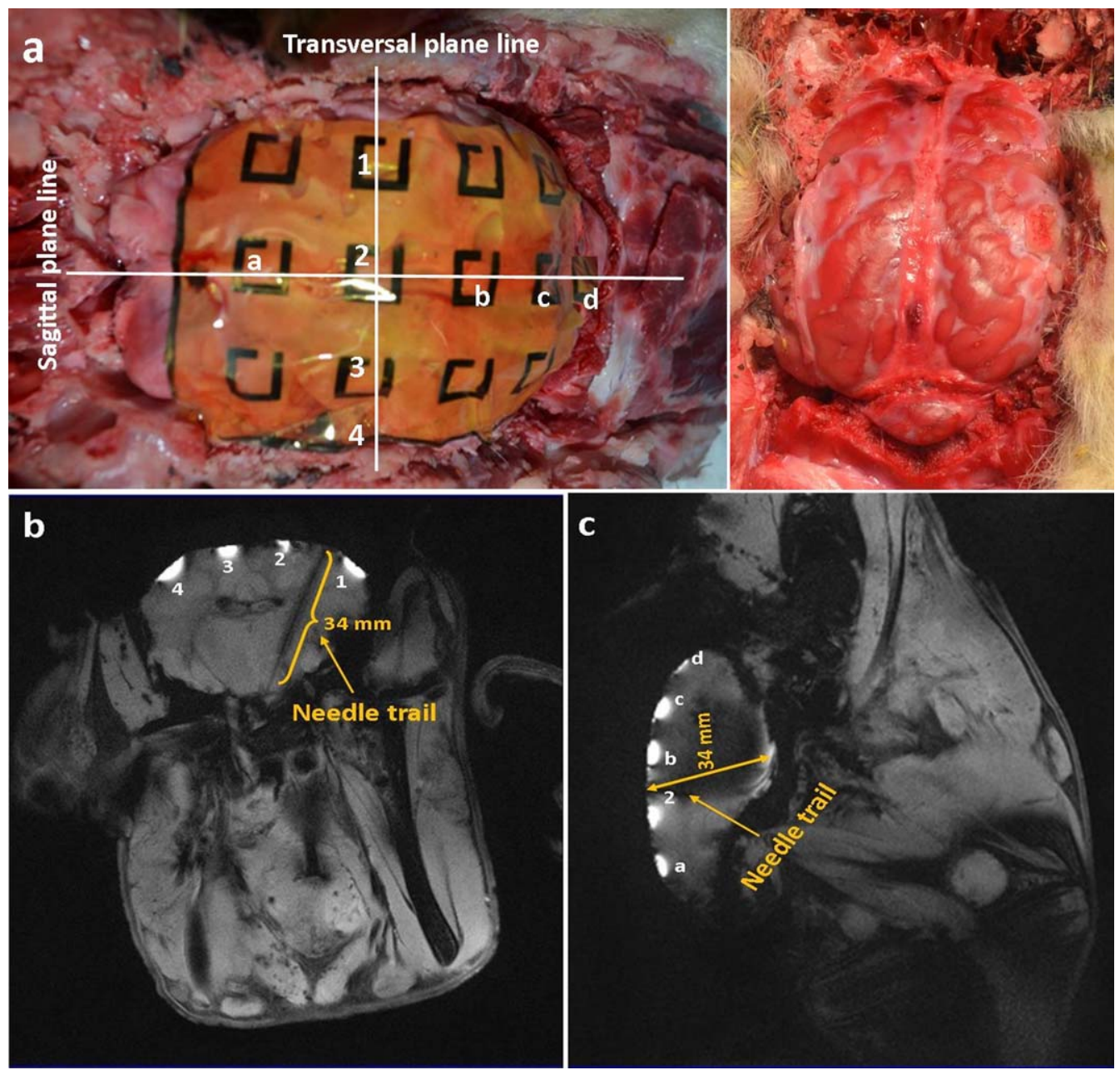

FIG. 7. Ex vivo marker study through the brain of a sheep. a: An array of flexible markers was set over the cerebral cortex of the brain. Every marker is indicated by a code. Excitations in transverse and sagittal plans are shown by the corresponding lines over the cerebral cortex. $\mathbf{b}$, $\mathbf{c}$ : Transverse and sagittal $\mathrm{T}_{2}^{*}$-weighted GRE images of the array of markers over the brain, along with an inserted nonmagnetic needle, were achieved. Each marker is denoted by its corresponding code in the MR images. The position of the needle with respect to the tissue and markers is evident.

maximum signal intensity via coupling to RF coils. However, the marker provides strong coupling efficiency even with a large misalignment from its normal axis.
An ex vivo MR experiment on a cadaver demonstrated the guidance of a needle using the intersection of the coronal and sagittal planes in MRI images. The clinical
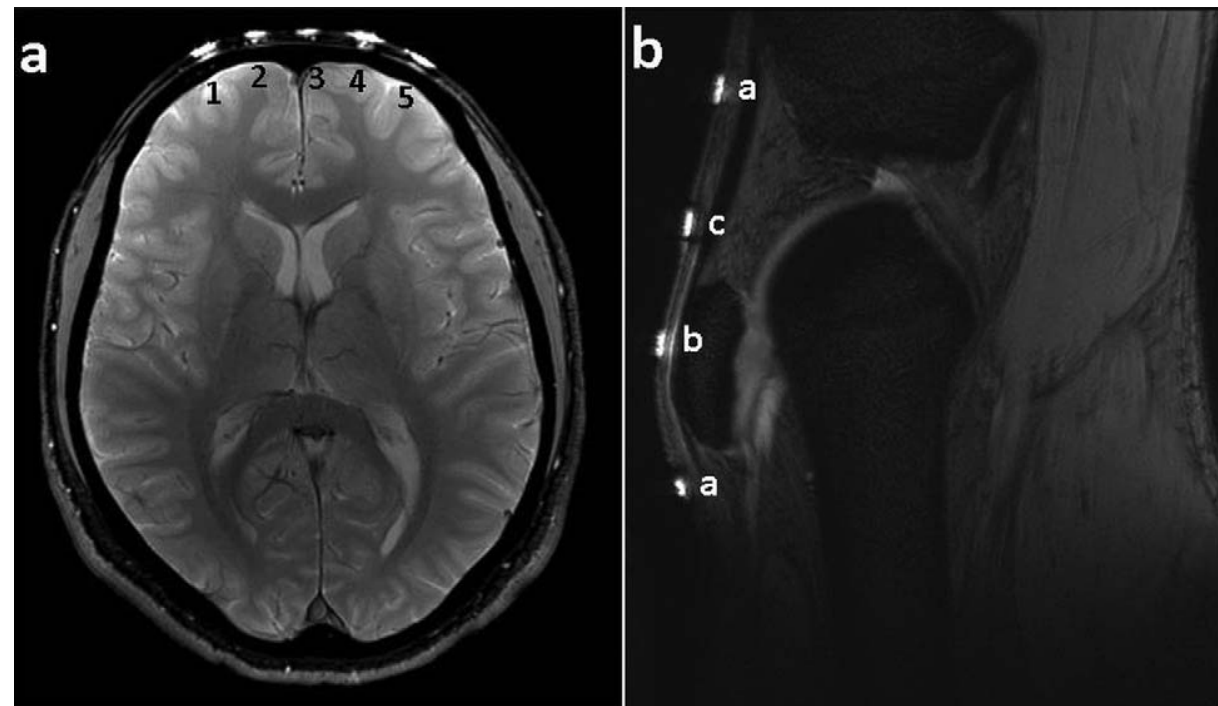

FIG. 8. Anatomic tissue localization using our passive RF-resonator markers placed over the human head (a) and the human knee (b). Resulting $\mathrm{T}_{2}^{*}$-weighted GRE images, transverse view of the head, and sagittal view of the knee provide useful information about the position of each marker with respect to the anatomic background. 
FIG. 9. Clinical feasibility of biopsy needle guidance using the resonator markers under a 3T MRI scanner. An array of the markers $(2 \times 2)$ was fixed over a patient's head containing a mass. a: Position of the mass with respect to the markers is clearly indicated on $\mathrm{T}_{1}$-weighted GRE images. b: After the positions of the markers and needle insertion point were graphically fiducial-marked, the needle insertion procedure was conducted gradually. $\mathrm{T}_{1}$-weighted GRE images show the position of the biopsy needle inside the brain close to the mass.

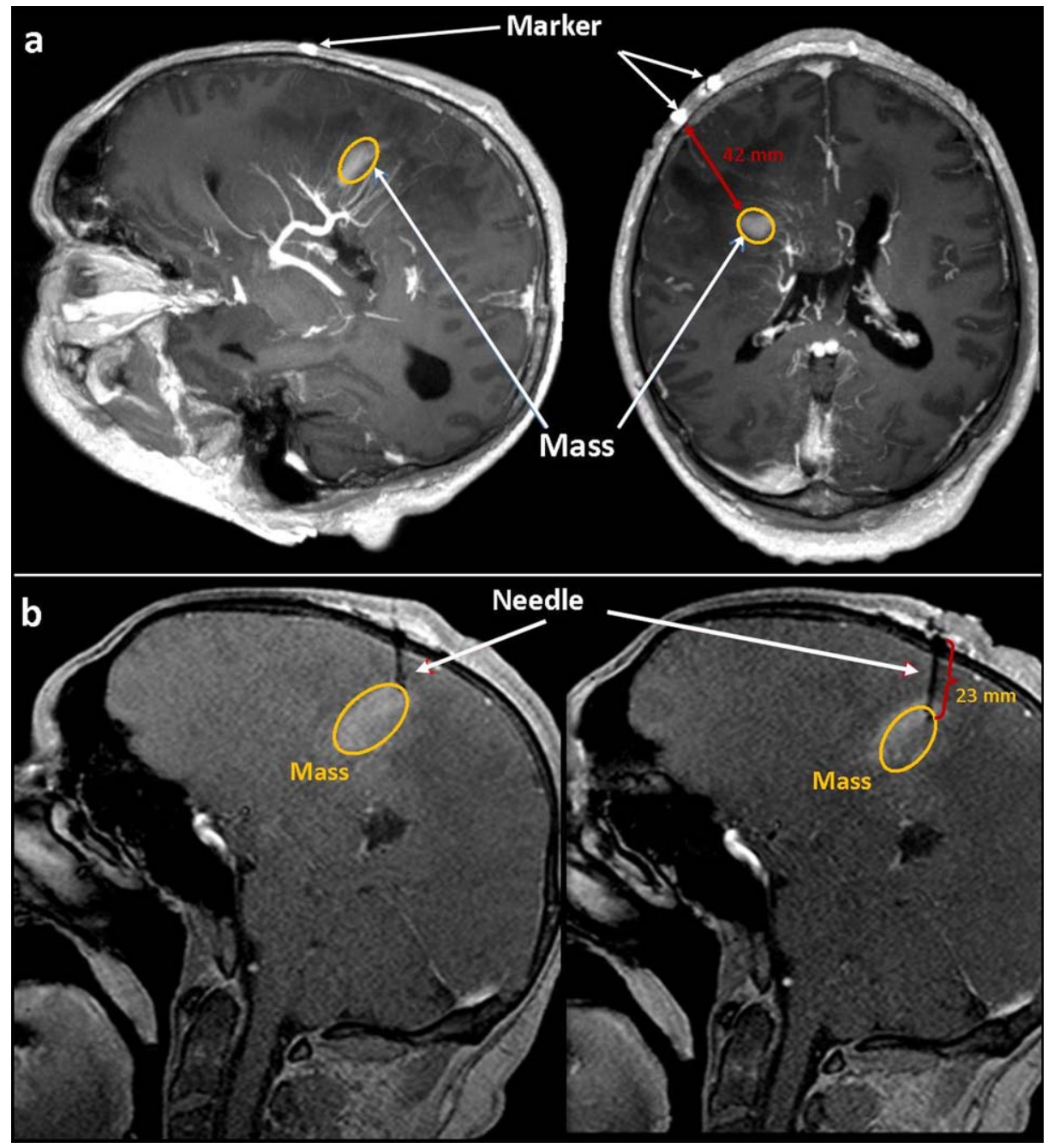

feasibility of the proposed resonator marker was also demonstrated in this study in guiding a biopsy needle. A patient with a mass in the brain was subjected to an MR experiment to mark the position of the mass with respect to the background and markers. The approximate insertion point of the needle was estimated by the intersection of the marker-bearing coronal and sagittal-plane MRI images. Therefore, ex vivo and human experiments were implemented to show the standard clinical performance of these markers. The experimental results indicated that this resonator can be freely and easily used for tissue-marking purposes in MRI on various body surfaces.

Magnetic resonance interventional catheter tracking systems using a modified version of these types of RF passive resonators remain an important future work. These would be used for determining the correct position of the intervention device inside the body. Using thin-film microwave technology, it is possible to miniaturize the proposed structure for purposes of implantation.

\section{CONCLUSIONS}

An ultra-thin, flexible, and passive RF-based resonator marker was successfully tested with phantom experiments.
Its performance was also shown in the cerebral cortex of an animal cadaver as well as in in vivo head and knee human experiments for fiducial marking purposes. The resonator marker enhances the signal in its vicinity, which results in high marker-to-background contrast, and consequently better MR visualization. This technique may inspire novel approaches in interventional MR-guided procedures.

\section{ACKNOWLEDGMENTS}

We gratefully acknowledge Dr. Gamze Aykut, Department of Molecular Biology and Genetics, Bilkent University (Ankara, Turkey), and Dr. Cagdas Oto, Faculty of Veterinary Medicine, Ankara University (Ankara, Turkey), for their assistance in the ex vivo experiments, and the UMRAM staff for providing technical support.

\section{REFERENCES}

1. Yang X, Atalar E. Intravascular MR imaging-guided balloon angioplasty with an MR imaging guide wire: feasibility study in rabbits. Radiology 2000;12:75-78.

2. Moche M, Trampel R, Kahn T, Busse H. Navigation concept for MR image-guided intervention. JMRI 2008;27;276-291.

3. Ashvin G, Merdim S, Robert L, Anthony F. Robust automatic rigid registration of MRI and X-ray using external fiducial markers for XFM-guided interventional procedures. Med Phys 2011;38:125-141. 
4. Lufkin R, Teresi L, Chiu L, Hanafee W. A technique for MR-guided needle placement. Am J Roentgenol 1988;151:193-196.

5. Masamune K, Kobayashi E, Masutani Y, et al. Development of an MRI-compatible needle insertion manipulator for stereotactic neurosurgery. J Image Guided Surg 1995;4:242-248.

6. Parker C, Damyanovich A, Haycocks T, Haider M, Bayley A, Catton C. Magnetic resonance imaging in the radiation treatment planning of localized prostate cancer using intra-prostatic fiducial markers for computed tomography co-registration. Radiother Oncol 2003;66:217224.

7. Bakker C, Hoogeveen R, Weber J, Vaals J, Viergever M, Mali W. Visualization of dedicated catheters using fast scanning techniques with potential for MR-guided vascular interventions. Magn Reson Med 1996;36:816-820.

8. Rubin DL, Ratner AV, Young SW. Magnetic susceptibility effects and their application in the development of new ferromagnetic catheters for magnetic resonance imaging. Invest Radiol 1990;25:1325-1332.

9. Koechli VD, McKinnon GC, Hofmann E, von Schulthess GK. Vascular interventions guided by ultrafast MR imaging evaluation of different materials. Magn Reson Med 1994;31:309-314.

10. Seppenwoolde JH, Viergever MA, Bakker CJ. Passive tracking exploiting local signal conservation: the white marker phenomena. Magn Reson Med 2003;50:784-790.

11. Quick HH, Zenge MO, Kuehl H, Kaiser G, Aker S, Massing S, Bosk S, Ladd ME. Interventional magnetic resonance angiography with no string attached: wireless active catheter visualization. Magn Reson Med 2005;53:446-455.

12. Zanchi MG, Venook R, Pauly JM, Scott GC. An optically coupled system for quantitative monitoring of MRI-induced RF currents into long conductors. IEEE Trans Med Imaging 2010;29:169-178.

13. Erturk MA, El-Sharkawy A-MM, Bottomley PA. Interventional loopless antenna at 7 T. Magn Reson Med 2012;68:980-988.

14. Etezadi-Amoli M, Stang P, Kerr A, Pauly J, Scott G. Interventional device visualization with toroidal transceiver and optically coupled current sensor for radiofrequency safety monitoring. Magn Reson Med 2015;73:1315-1327.

15. Wang W, Dumoulin CL, Viswanathan AN, et al. Real-time active MRtracking of metallic stylets in MR-guided radiation therapy. Magn Reson Med 2015;73:1803-1811.

16. Bernstein MA, Shu Y, Elliott A M. RINGLET motion correction for 3D MRI acquired with the elliptical centric view order. Magn Reson Med 2003;50:802-812.

17. Yunhong Shu Y, Elliott AM, Riederer SJ, Bernstein MA. Motion correction properties of the shells k-space trajectory. Magn Reson Imaging 2006;24:6:739-749.

18. Thormer G, Garnov N, Mocke M, Haase J, Kahn T, Busse H. Simultaneous 3D localization of multiple MR- visible markers in fully reconstructed MR images: proof-of-concept for subsecond position tracking. Magn Reson Imaging 2012;30:371-381.

19. Unal O, Korosec FR, Frayne R, Strother CM, Mistretta CA. A rapid 2D time-resolved variable-rate $k$-space sampling MR technique for passive catheter tracking during endovascular procedures. Magn Reson Med 1998;40:356-362.

20. Xinga X, Zhangb B, Wanga X, Liua F, Shib D, Chengd Y. An imagingbiopsy strategy for colorectal tumor reconfirmation by multipurpose paramagnetic quantum dots. Biomaterials 2015;48:16-25.

21. Unal O, Li J, Yu H, Strother CM. In vitro and in vivo evaluation of MR visible coated-devices. In Proceedings of the 10th Annual Meeting of ISMRM, Honolulu, Hawaii, USA, 2002. p. 2219.

22. Dahnke H, Liu W, Herzka D, Frank JA, Schaeffter T. Susceptibility gradient mapping (SGM): a new postprocessing method for positive contrast generation applied to superparamagnetic iron oxide particle (SPIO)-labeled cells. Magn Reson Med 2008;60:595-603.

23. Richardson OC, Bane O, Scott ML, Tanner SF, Waterton JC, Sourborn SP, Carrol TJ, Buckley DL. Gadofosveset-based biomarker of tissue albumin concentration: technical validation in vitro and feasibility in vivo. Magn Reson Med 2015;73:244-253.
24. Klemm P, Floyd W, Smiles D, Fréchet J, Raymond K. Improving T1 and T2 magnetic resonance imaging (MRI) contrast agents through the conjugation of an esteramide dendrimer to high water coordination Gd(III) hydroxypyridinone (HOPO) complexes. Contrast Media Mol Imaging 2012;7:95-99.

25. Atalar E, Bottomley PA, Ocali O, Correia LC, Klemen MD, Lima JA, Zerhouni EA. High resolution intravascular MRI and MRS by using a catheter receiver coil. Magn Reson Med 1996;36:596-605.

26. Ocali O, Atalar E. Intravascular magnetic resonance imaging using a loopless catheter antenna. Magn Reson Med 1997;37:112-118.

27. Viswanathan AN, Cormack R, Holloway CL, Tanaka C, O’Farrell D, Devlin PM, Tempany C. Magnetic resonance-guided interstitial therapy for vaginal recurrence of endometrial cancer. Int J Radiat Oncol Biol Phys 2006;66:91-99.

28. Fandrey S, Weiss S, Müller J. A novel active MR probe using a miniaturized optical link for a 1.5-T MRI scanner. Magn Reson Med 2012; 67:148-155.

29. Wildermuth S, Dumoulin CL, Pfammatter T, Maier SE, Hofmann E, Debatin JF. MR-guided percutaneous angioplasty: assessment of tracking safety, catheter handling and functionality. Cardiovasc Intervent Radiol 1998:21:404-410.

30. Nitz WR, Oppelt A, Renz W, Manke C, Lenhart M, Link J. On the heating of linear conductive structures as guide wires and catheters in interventional MRI. J Magn Reson Imaging 2001:13:105-114.

31. Weiss S, Kuehne T, Brinkert F, Krombach G, Katoh M, Schaeffter T, Guenther RW, Buecker A. In vivo safe catheter visualization and slice tracking using an optically detunable resonant marker. Magn Reson Med 2004;52:860-868.

32. Burl M, Coutts GA, Young IA. Tuned fiducial markers to identify body locations with minimal perturbation of tissue magnetization. Magn Reson Med 1996;36:491-493.

33. Quick HH, Ladd ME, Nanz D, Mikolajczyk KP, Debatin JF. Vascular stents as RF antennas for intravascular MR guidance and imaging. Magn Reson Med 1999;42:738-745.

34. Celik H, Atalar E. Reverse polarized inductive coupling to transmit and receive radiofrequency coil arrays. Magn Reson Med 2012;67:446-456.

35. Marques R, Martin F, Sorolla. Metamaterials with negative parameters: theory, design and microwave applications. New York: John Wiley; 2008. p 315.

36. Freire MJ, Lopez MA, Meise F, Algarin JM, Jakob PM, Bock M, Marques R. A broadside-split-ring resonator-based coil for MRI at $7 \mathrm{~T}$. IEEE Trans Med Imaging 2013;32:6:1081-1084.

37. Corea RJ, Flynn AM, Lechêne B, Scott G, Reed DG, Shin PJ, Lustig M, Arias AC. Screen-printed flexible MRI receive coils. Nature Communications 2016;7:10839.

38. Farcich NJ, Salonen J, Asbeck PM. Single-length method used to determine the dielectric constant of polydimethylsiloxane. IEEE Trans Microw Theory 2008;12:2963-2971.

39. Constantinides CD, Atalar E, McVeigh ER. Signal-to-noise measurements in magnitude images from NMR phased arrays. Magn Reson Med 1997;38:5:852-557.

40. Hornak J, Szumowski J, Bryant R. Magnetic field mapping. Magn Reson Med 1987;6:158-163.

41. Acikel V, Atalar E. Modeling of radio-frequency induced currents on lead wires during MR imaging using a modified transmission line method. Med Phys 2011;38:6623-6632.

42. ASTM. Measurement of radio frequency induced heating on or near passive implants during magnetic resonance imaging. ASTM F2482$11 \mathrm{a} ; 2011$.

43. Hall WA, Liu H, Martin AJ, Pozza CH, Maxwell RE, Truwit CL. Safety, efficacy, and functionality of high-field strength interventional magnetic resonance imaging for neurosurgery. Neurosurgery 2000;46:632-641.

44. Dumoulin CL, Mallozzi RP, Darrow RD, Schmidt EJ. Phase-field dithering for active catheter tracking. Magn Reson Med 2010:63: 1398-1403. 